\title{
Development of a Simple System for the Determination of Arsenic after Hydride Generation Atomic Absorption Spectrophotometry
}

\author{
* MUMUNI, A.R. ; PEACOCK, C. J \\ Department of chemistry, University of Lancaster, Lancaster LAI 4YA, UK
}

\begin{abstract}
A simple and inexpensive hydride generation system that uses a pyrex boiling tube as a reduction vessel to hold the acidified sample into which $2 \mathrm{~cm}^{3}$ of $100-300 \mathrm{mg}$ sodium tetrahydroborate (III) solution is injected such that the excess molecular hydrogen evolved carries the generated arsine directly through the atomic absorption spectrophotometer nebuliser to a nitrogen-hydrogen entrained air flame for atomic absorption measurement is described. The average absorption by arsenic atoms generated from the total arsine evolved was measured by integrating the absorbance for 16 seconds. The use of the absorption signal integration mode was found to free the technique from most kinetic interferences, while the stripping of the arsine from solution reduces the potential for chemical interferences in the solution phase. Gas flow rates affected sensitivity markedly, but the optimal flow rates for nitrogen and hydrogen were standardised on $90 \mathrm{~cm}^{3} \mathrm{~s}^{-1}$ and $30 \mathrm{~cm}^{3} \mathrm{~s}^{-1}$ respectively as a compromise between lower flow-rates giving longer arsenic residence times and higher sensitivity, and higher flowrates giving a stiffer, less draught-sensitive flame. An optimum flame height of $6 \mathrm{~mm}$ above the burner produced sufficient atomization and low background absorption. Generally, sample volumes between $5-20 \mathrm{~cm}^{3}$ could be used in different acidic media within the concentration range $10 \%(\mathrm{v} / \mathrm{v})-50 \%(\mathrm{v} / \mathrm{v})$. The proposed system produced a significantly improved atomic absorption sensitivity of 25ng and a detection limit of (95\% confidence) of 5.2ng for arsenic. Both performance characteristics were found to be better by a factor $\geq 12$ and $\geq 27$ respectively than achieved with air-acetylene flames. The method is highly reproducible at trace levels having an overall precision of $1 \%$ for $0.5 \mu \mathrm{g}$ of arsenic. The upper limit of linearity of the response for atomic absorption measurements is about $0.7 \mu \mathrm{g}$. In use, the apparatus is fast and convenient to operate, enabling about forty determinations per hour. @ JASEM
\end{abstract}

Braman et al, (1972) introduced the sodium tetrahydroborate (III) acid reaction $\left(\mathrm{NaBH}_{4} /\right.$ acid) system as a better alternative to the first most frequently used metal/acid reaction of Holak (1969) to quantitatively generate volatile covalent hydrides for Atomic Absorption Spectrophotometric (AAS) measurement. Since then several workers have used the method of Braman et al, (1972) to develop expensive Hydride Generation (HG) accessory currently in the market. Inspite of the progress made in the development of the HG system and its advantages over the conventional method (by solution aspiration into the air-acetylene flame), many analytical/quality control laboratories are yet to adopt the $\mathrm{HG}$ system for hydride forming elements of Groups IV, V, and VI either due to cost implications, lack of expertise, or more importantly, the inability of analysts to take cognisance of the serious limitations of the airacetylene system in chemical analysis. These limitations include: poor atomic absorption sensitivity; poor detection limit; poor atomization efficiency; significant chemical interferences and severe flame absorption in the low ultraviolet region of the spectrum where most of the absorbance lines of hydride forming elements are located. These limitations all combine to introduce errors particularly at trace levels among which is a risk of concluding that a determinant is present when it is not or a risk of concluding that a determinant is absent when infact it is not. This explains why the quality of analytical data generated for covalent hydride elements using the air - acetylene flame method at trace levels have become suspect, inaccurate and unreliable.

Consequently, we were driven by the need to develop an operationally simpler, inexpensive and rapid HG system which can be used to minimize or improve on the serious limitations associated with the air-acetylene flame atomization technique for arsenic.In this study, arsenic was selected as the analyte considering its wide distribution throughout the biosphere and its significance as an environmental pollutant with no known beneficial biological function. Furthermore, because it is both toxic and carcinogenic (Hernberg, 1977) with adverse effects on the nervous system, cardiovascular system and the genetic system in man (Fowler et al 1979), the need for a simple and sensitive analytical system for detection of low level arsenic in a wide range of environmental and biological samples is axiomatic.It is hoped that chemists and analysts will take advantage of this simple HG accessory to improve on the quality of analytical output for arsenic in the course of their investigations.

* Corresponding author

Present Address: Department of Pure and Industrial Chemistry, University of Port Harcourt, PMB 5323, Port Harcourt, Nigeria. 
EXPERIMENTAL

\section{Hydride Generator Design}

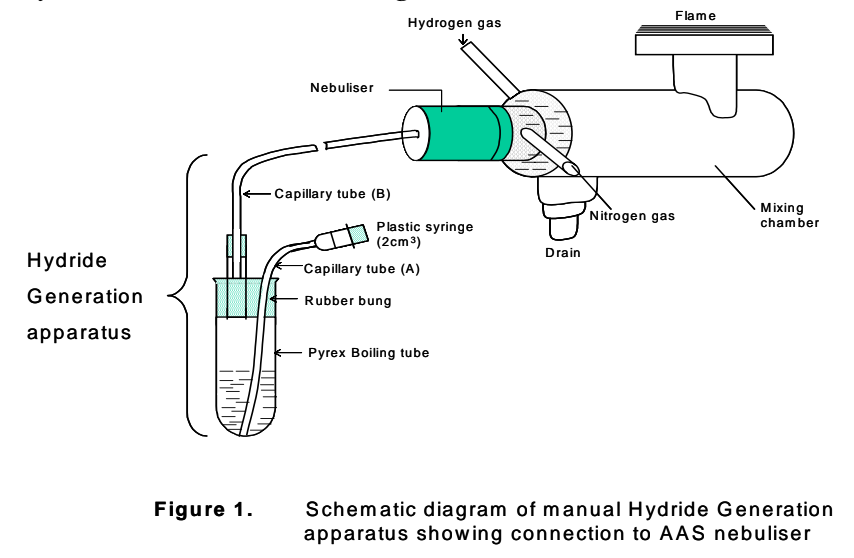

The Hydride Generator accessory utilises a $23 \mathrm{~mm}$ i.d., $150 \mathrm{~mm}$ long Pyrex boiling tube as the reduction vessel on to which is fitted a rubber bung which carries firstly a short length of $1 \mathrm{~mm}$ i.d plastic capillary (A) reaching to the bottom of the boiling tube down which sodium tetrahydroborate (111) solution may be injected from a $2 \mathrm{~cm}^{3}$ plastic syringe and secondly, a glass tube connected by a small rubber bung and a length of $1 \mathrm{~mm}$ i.d. plastic capillary (B) to the spectrophotometer nebuliser inlet (figure 1). The HG accessory is placed in a $400 \mathrm{~cm}^{3}$ Pyrex beaker for support.

\section{Reagents}

Analytical - reagent grade chemicals and deionised water were used throughout the experimentation except for the tetrahydroborate (III)which was obtained as reagent grade powder from Aldrich Chemical company Inc.

$1000 \mu \mathrm{g} \mathrm{cm}^{-3}$ arsenic (III) standard solutions were prepared by dissolving 1.3203 g arsenic (III) oxide in a minimum volume of $20 \% \mathrm{w} / \mathrm{v}$ sodium hydroxide followed by neutralization with nitric acid and dilution to 1 litre of solution. Working standard solutions were prepared as required by serial dilution of the $1000 \mu \mathrm{g} \mathrm{cm}^{-3}$ arsenic (III) standard stock solution with acid of appropriate concentration.

Sodium tetrahydroborate reducing solutions were prepared as required by dissolving the reagent grade material in deionised water in which one pellet of sodium hydroxide was dissolved for every $20 \mathrm{~cm}^{3}$ of the solution to prevent hydrolysis. Throughout this study it was used as an aqueous solution rather than as a solid in order to avoid problems that might arise from the employment of a fast heterogeneous reaction.

\section{Instrumentation}

Atomic absorption measurements in the premixed nitrogen-hydrogen entrained-air flame were made with an Instrumentation Laboratory, IL 251, double beam Atomic Absorption Spectrophotometer equipped with a single slot, $10 \mathrm{~cm}$ boling-type burner head and a Rank-Hilger arsenic hollowcathode lamp.

Table 1. Instrumental parameters and solution conditions for the optimisation of operational parameters for arsenic.

\begin{tabular}{|l|l|l|l|l|l|l|l|}
\hline $\begin{array}{l}\text { Wave } \\
\text { length } \\
(\mathrm{nm})\end{array}$ & $\begin{array}{l}\text { Lamp } \\
\text { current } \\
(\mathrm{mA})\end{array}$ & $\begin{array}{l}\text { Slit width } \\
\text { um/SBW } \\
(\mathrm{nm})\end{array}$ & $\begin{array}{l}\text { Nebuliser } \\
\text { flow rate }\end{array}$ & $\begin{array}{l}\mathrm{NaBH}_{4} \\
\text { Conc } \\
\mathrm{m} / \mathrm{v}(\%)\end{array}$ & $\begin{array}{l}\text { Optimum } \\
\text { amount of } \\
\text { analyte in } \\
\text { cell }-\mu \mathrm{g}\end{array}$ & $\begin{array}{l}\text { Acid addition to } \\
\text { cell }\end{array}$ & $\begin{array}{l}\text { Total vol } \\
\text { in cell } \\
\left(\mathrm{cm}^{3}\right)\end{array}$ \\
\hline 193.7 & 5 & $320 / 1$ & Maximum & 15 & 0.5 & $\begin{array}{l}10 \mathrm{~cm}^{3} \\
10 \%(\mathrm{v} / \mathrm{v}) \mathrm{HCl}\end{array}$ & 10 \\
\hline
\end{tabular}


Procedure for optimising operational parameters for the determination of arsenic after hydride Generation Atomic Absorption Spectrophotometry:

Using the instrumental parameters and solution conditions in Table 1 and the (Recommended Procedure for Generation and Measurement of the hydride), operational parameters which consisted of input parameters (flame height, gas flow rates, acidity, volume of sample solution, sodium tetrahydroborate (III) concentration) and output parameters (linearity of response, precision and reproducibility, limit of detection) were determined as follows:

Effect of hydrogen flow rate and flame height The optimal flame conditions in the premixed nitrogen-hydrogen entrained air flame were determined by measuring the atomic absorption signals for hydrogen flow rates of $15 \mathrm{~cm}^{3} \mathrm{~s}^{-1}$ (2scfh) to $60 \mathrm{~cm}^{3} \mathrm{~s}^{-1}$ (8scfh) at varying flame heights of $2-$ $24 \mathrm{~mm}$, while the total flow rate of nitrogen was kept constant at $90 \mathrm{~cm}^{3} \mathrm{~s}^{-1}$ (12scth). For all measurements, the burner control was used to move the flame vertically relative to the light path of the optical system.

Effect of carrier Gas flow rate

To find the optimal flow rate of carrier gas, the flame height and hydrogen flow rate were kept constant at $6 \mathrm{~mm}$ (above the burner) and $30 \mathrm{~cm}^{3} \mathrm{~s}^{-1}$ (4scfh) respectively, while the atomic absorption signals were measured for nitrogen flow rates of $75 \mathrm{~cm}^{3} \mathrm{~s}^{-1}$ (10scfh) $-180 \mathrm{~cm}^{3} \mathrm{~s}^{-1}$ (24scfh).

\section{Effect of volume of solution}

The effect of the volume of solution in the boiling tube of the HG apparatus on the response for a constant absolute mass of arsenic was studied for sample volumes varying between 5 and $20 \mathrm{~cm}^{3}$.

Effect of amount of sodium tetrahydroborate (III)

The effect of sodium tetrahydroborate (III) in the concentration range $1-15 \% \mathrm{~m} / \mathrm{v}$ on sensitivity to arsenic was examined.

Effect of acidity and type of acid

The relative response of arsenic was studied in a number of acid media by varying the acid concentrations between $1 \%(\mathrm{v} / \mathrm{v})$ to $50 \%(\mathrm{v} / \mathrm{v})$ in order to establish conditions for optimum sensitivity and so consider the effect of acid media that were likely to be residual from sample digestion procedures.

\section{Linearity}

The linearity of response was established by analysing standards containing between 0.05 $1.5 \mu \mathrm{g}$ of arsenic.

Precision and Reproducibility

Ten replicate determinations in each case were carried out using the optimum reagent conditions in Table 1.

\section{Limit of detection}

For each measurement, a standard solution containing 20ng of arsenic in $10 \mathrm{~cm}^{3}$ of $10 \%(\mathrm{v} / \mathrm{v})$ hydrochloric acid was analysed using $2 \mathrm{~cm}^{3}$ of $5 \%$ $(\mathrm{m} / \mathrm{v})$ sodium tetrahydroborate (III) in order to minimize dilution effects of the analyte at such low concentration as well as lessen the flame disturbance and blank from arsenic in this reagent. Eleven and ten alternate determinations of the reagent blank and analyte were analysed.

Unless otherwise stated, all input and output parameters were determined in triplicate throughout the experimentation.

Recommended Procedure for the Generation and Measurement of the Hydride

Prior to the actual determinations, the atomic absorption spectrophotometer should be allowed to warm up under the instrument operating conditions in Table 1 and then adjusted to zero absorbance by simply passing air through it, after which two blank determinations are made for each sample. For each determination, the rate of injection of the sodium tetrahydroborate (III) solution should be carefully controlled: (i) to ensure complete reaction of the analyte and evolution of all the arsine within the integration time and (ii) to prevent the transmittance of the arsine evolved from falling into the nonlinear region of the instrument intensity meter. Aliquots of arsenic standard to be analysed are quantitatively transferred into the boiling tube of the hydride generation accessory and acid diluent of the appropriate concentration is added to bring the total volume of solution to about $10 \mathrm{~cm}^{3}$. The sample tube is then tightly stoppered with the rubber bung carrying a plastic capillary tube inlet (A) and outlet (B) (Figure 1). The exit capillary, B, of the hydride generation accessory is connected directly to the nebulizer inlet of the AAS and $2 \mathrm{~cm}^{3}$ of sodium tetrahydroborate (III) solution of the required concentration injected through capillary tube inlet A. In acid solution, the sodium tetrahydroborate (III) reacts with As(III) to form arsine as given in the equation below: 
and also excess molecular hydrogen is produced in the process as shown below::

$$
\mathrm{BH}_{4}+3 \mathrm{H}_{2} \mathrm{O}+\mathrm{H}^{+} \longrightarrow \mathrm{H}_{3} \mathrm{BO}_{3}+4 \mathrm{H}_{2} \text { (excess) }
$$

The generated hydride is then swept from the solution directly into a nitrogen - hydrogen entrained air flame by means of the excess molecular hydrogen generated during the reduction process. Then the absorbance, that is, absorbance averaged over $16 \mathrm{~s} \times 1000$ is integrated over a period of 16 seconds and recorded. In this study, the beginning of the absorption signal was observed in less than a second after injection of the reductant and within twelve seconds the reduction of the analyte to its volatile hydride had been completed.

It is pertinent to note that whilst examining the operating conditions, we found that the method of measuring response by peak height measurement was inadequate due to kinetic interferences but the integration mode was not. As a consequence, the use of the integration mode was adopted and it was found convenient to integrate the absorbance from the whole of the arsine evolved. This produced satisfactory results for between sample reproducibility to better than $1 \%$ at $0.5 \mu$ g of arsenic per sample (Figure 8).

After evolution has ceased, the syringe is disconnected and wiped with a soft tissue paper. At this stage, residual sodium tetrahydroborate (III) solution that might squirt is caught by holding the syringe nozzle in a tissue. The rubber bung is then disconnected from the sample tube and wiped, and is then ready for the next sample.

\section{RESULTS AND DISCUSSION}

Effect of gas flow rates and flame height : Figures 2 and 3 show the effect of gas flow rates and flame height on sensitivity for arsenic. The absorption signals increased with decrease in hydrogen flow rates. Conversely, increased hydrogen flow rates normally result in an increase in both the flame temperature and pressure as well as the rise velocity of the premixed flame gases. Hence, all these variables combine to cause dilution effect of the free analyte atoms thereby yielding reduced sensitivity at high hydrogen flow rates as observed in this study. Similarly, lower nitrogen flow rates also increased the sensitivity while higher nitrogen flow rates resulted in decreased sensitivity partly due to dilution and partly due to the shortened residence time of the free analyte atoms in the flame atom cell. For optimum gas flow rates, we standardised on $30 \mathrm{~cm}^{3} \mathrm{~s}^{-1}$ for hydrogen and $90 \mathrm{~cm}^{3} \mathrm{~s}^{-1}$ for nitrogen as a compromise between lower flow rates giving longer arsenic residence times and higher sensitivity, and higher flow rates giving a stiffer, less draught sensitive flame.

Flame height also affected the sensitivity markedly: increase in the flame height resulted in increased sensitivity until an optimum height was obtained beyond which sensitivity decreased markedly. The height of maximum absorption marks the level where the increased atomization with height is just balanced by the rate of decrease in the concentration of the free atoms through dilution by the flame gases. A flame height of $6 \mathrm{~mm}$ above the burner was chosen as a compromise because at this height sufficient atomization was achieved and the flame exhibited a considerably low background absorption. The relative maximum flame height used also indicate the stability of the hydride to decomposition.

Effect of volume of solution: The results of the effect of this variable on the sensitivity of the hydride revealed that over the studied range of sample sizes, the response was almost invariant (Figure 4). This can be attributed to the design of the apparatus as well as the efficiency of using excess hydrogen generated during the reduction stage in the flushing system. In the course of this study, we also found that for solution volumes below $5 \mathrm{~cm}^{3}$ mixing gave rise to a slightly variable response while above $20 \mathrm{~cm}^{3}$ the volume was too large for the boiling tube. The use of larger boiling tubes proved unsuccessful because the arsine evolution was then too slow to be completed in 16 seconds. In view of this, $10 \mathrm{~cm}^{3}$ sample volume was chosen as standard.

Effect of amount of sodium tetrahydroborate(III): The results obtained (figure 5) show that as the amount of sodium tetrahydroborate (III) solution injected into the acidified sample was increased, the response first rose, then reached a plateau for amounts between $100-300 \mathrm{mg}$. However, we also found that too high a concentration increased solution viscosity unfavourably and this gave rise to unreproducible and less sensitive determination because it reduces the acid concentration to below the optimum level for the reduction. In view of this, the amount of sodium tetrahydroborate (III) used throughout this study was kept within the optimum concentration range established 


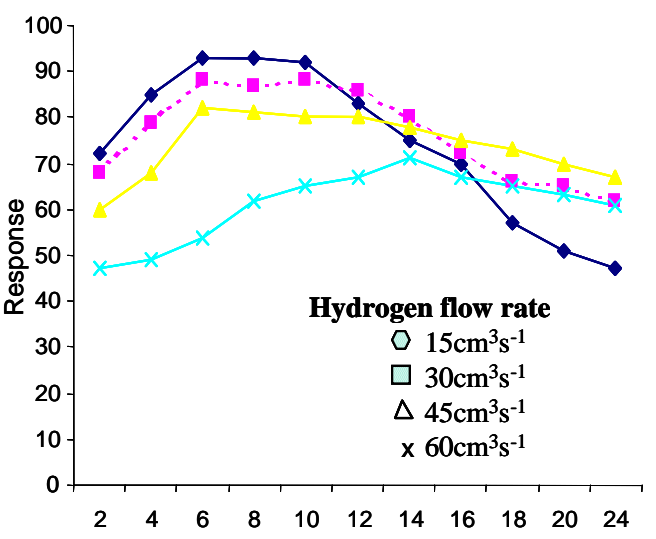

Flame Height, $\mathbf{m m}$

Figure 2: Effect of hydrogen flow rate and flame height on sensitivity to arsenic

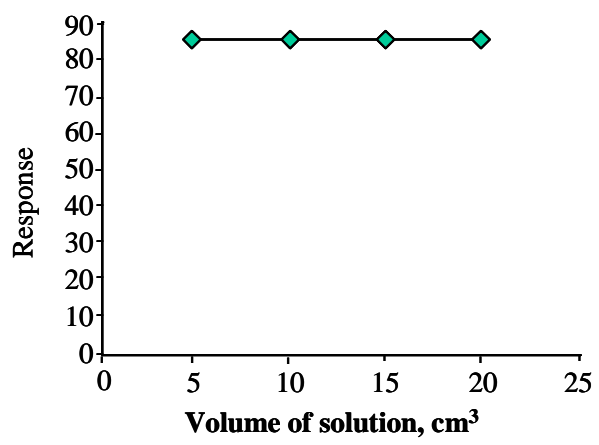

Figure 4: Effect of volume of solution on sensitivity to arsenic

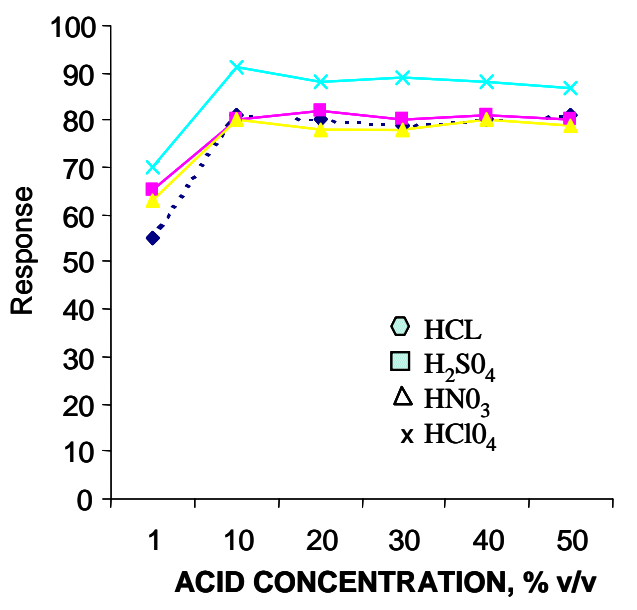

Figure 6: Effect of acidity on the analytical response for arsenic

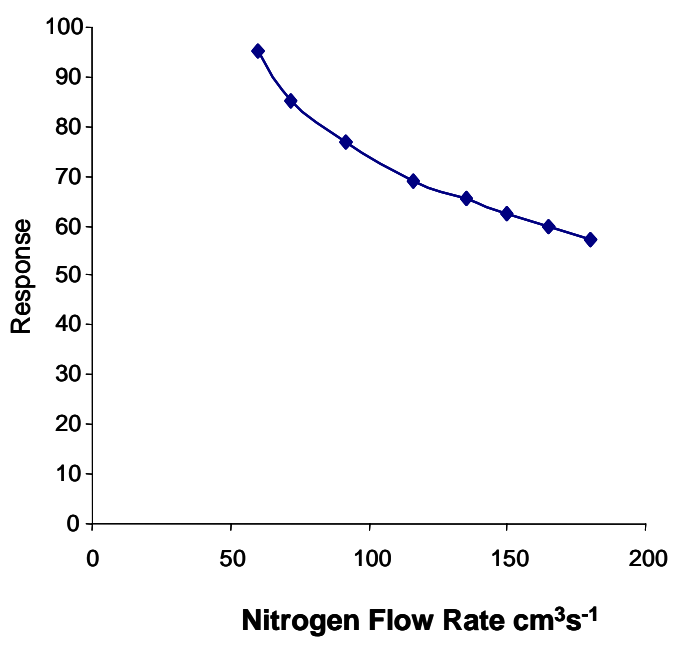

Figure 3: Effect of carrier gas flow rate on the determination of arsenic

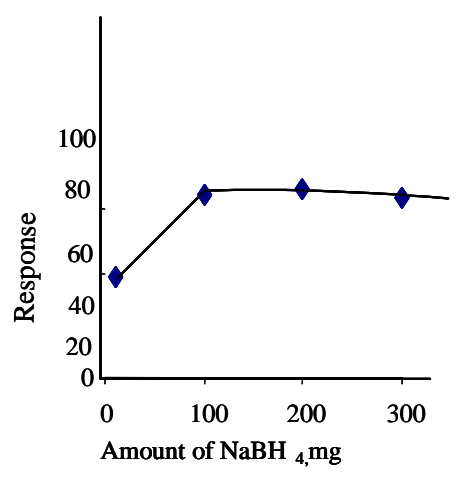

Figure 5: Effect of sodium tetrahydroborate (III) on sensitivity to the determination of arsenic

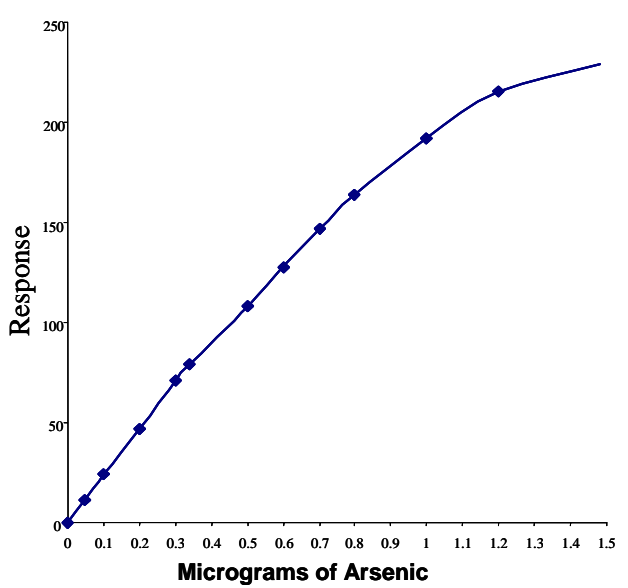

Figure 7: Calibration curve for arsenic 
Effect of acidity and type of acid: Figure 6 presents the response of arsenic as a function of acidity or hydrogen ion concentration. The results show that the response were independent of acid in the range $10 \%(\mathrm{v} / \mathrm{v})-50 \%(\mathrm{v} / \mathrm{v})$ irrespective of acid. These results compare well with those of Thomson and Thomerson (1974) and Nadkarni (1982).

Generally, the results of these investigations show that provided there is more than the theoretical amount of acid present for complete reduction with sodium tetrahydroborate (III), the total acidity or type of acid is immaterial for the determination of arsenic. This insensitivity to type or concentration of acid makes the digestion of the samples much easier and there is no need to evaporate off large volumes of acid with possible consequent losses of the analyte, and the acid regime most convenient for sample decomposition may be used.

Furthermore, the results obtained with the use of perchloric acid show that the system has potential applications in the analysis of a variety of biological and environmental samples in which perchloric acid is likely to be present in the residual solutions after digestion.

Linearity and Atomic Absorption Sensitivity: Figure 7 shows that the upper limit of linearity of the response for atomic absorption measurements using our system was about $0.7 \mu$ g beyond which both linearity and reproducibility are lost. Though we found out that linearity could be extended to a limit of about $1 \mu \mathrm{g}$ if the sodium tetrahydroborate (III) solution could be added slowly at a more or less constant rate of about 10 seconds.

Rather than use the definition of sensitivity common to practising atomic absorption spectroscopists, i.e., the concentration of analyte, in $\mu_{\mathrm{gcm}}{ }^{-3}$ which will produce $1 \%$ absorption $(0.0044 \mathrm{~A})$, the authors prefer the use of the "slope" of the linear portion of the analytical calibration curve and the mathematical definition of atomic absorption sensitivity, i.e., $(\underline{0.0044})_{\mathrm{T}}=99 \%$, where $m$ is the slope to obtain a value of $25 n$.

Comparatively, this much-improved atomic absorption sensitivity is significantly better than achieved with air-acetylene flame systems reported by different AAS instrument manufacturers (Table 4).

Precision and reproducibility: Table 2 shows the precision of the method under normal conditions giving a relative standard deviation of about $1.0 \%$ for $0.5 \mu \mathrm{g}$ of arsenic. Furthermore, it could be observed that the precision does not deteriorate to any significant extent for the overall determination as evidenced in Figure 8 which shows the high reproducibility of ten separate analyses of arsenic using the same instrumental and solution conditions as for the precision measurement.

Table 2 Precision and Reproducibility test for Arsenic

\begin{tabular}{|l|l|l|l|}
\hline Amount of Arsenic, $\mu \mathrm{g}$ & Average absorbance over 16s x 1000 & Mean & $\begin{array}{l}\text { Relative Standard } \\
\text { deviation, \% }\end{array}$ \\
\hline 0.5 & $85,87,85,86,86,85,85,85,84,85$ & 85.3 & 1.0 \\
\hline
\end{tabular}

Figure 8: Typical recorder tracings of reproducibility of Arsenic for 10 replicate standard solutions. (1division = 12 seconds) 
Table 3 Limit of detection for arsenic

\begin{tabular}{|c|c|c|c|}
\hline & Average Absorbance & Mean & Standard deviation \\
\hline Blank - - - & $\begin{array}{lllllllllll}19 & 20 & 20 & 19 & 21 & 21 & 19 & 20 & 21 & 21 & 20\end{array}$ & 20.1 & 0.8 \\
\hline 20ng of arsenic & $\begin{array}{llllllllll}31 & 29 & 30 & 34 & 33 & 31 & 34 & 30 & 36 & 33\end{array}$ & 32.1 & 2.2 \\
\hline Net Response: & 12.0 (standard deviation 2.3 ) & & \\
\hline
\end{tabular}

Table 4 Performance characteristics of air - acetylene AAS methods and the proposed method for determination of arsenic after reduction to the hydride by sodium tetrahydroborate (III)

\begin{tabular}{|c|c|c|c|c|c|c|c|}
\hline \multirow[b]{3}{*}{ PARAMETER } & \multicolumn{7}{|c|}{ INSTRUMENT MODEL/FLAME AAS METHOD } \\
\hline & \multicolumn{2}{|c|}{ IL 451} & \multicolumn{2}{|c|}{ Fisher 850} & \multicolumn{2}{|c|}{$\begin{array}{l}\text { Thermo Jarrel Ash } \\
\text { SH } 22\end{array}$} & \multirow{2}{*}{$\begin{array}{l}\text { This } \\
\text { Work } \\
\mathrm{N}_{2}-\mathrm{H}_{2}\end{array}$} \\
\hline & Air- $\mathrm{C}_{2} \mathrm{H}_{2}$ & $\begin{array}{l}\mathrm{N}_{2}-\mathrm{H}_{2} \\
\text { AVA }\end{array}$ & Air- $\mathrm{C}_{2} \mathrm{H}_{2}$ & $\begin{array}{l}\mathrm{N}_{2}-\mathrm{H}_{2} \\
\text { AVA }\end{array}$ & Air- $\mathrm{C}_{2} \mathrm{H}_{2}$ & $\begin{array}{l}\mathrm{N}_{2}-\mathrm{H}_{2} \\
\text { AVA }\end{array}$ & \\
\hline AA sensitivity & $0.4 \mu \mathrm{gcm}^{-3}$ & NA & $0.3 \mu \mathrm{gcm}^{-3}$ & NA & $0.4 \mu \mathrm{gcm}^{-3}$ & 4ng & $25 n g$ \\
\hline $\begin{array}{l}\text { Upper limit of } \\
\text { linearity }\end{array}$ & $20 \mu \mathrm{gcm}^{-3}$ & NA & $100 \mu \mathrm{gcm}^{-3}$ & NA & $20 \mu \mathrm{cm}^{-3}$ & 130ng & 700ng \\
\hline $\begin{array}{ll}\text { Limit } & \text { of } \\
\text { Detection } & \end{array}$ & NA & NA & $0.2 \mu \mathrm{gcm}^{-3}$ & NA & $0.14 \mu \mathrm{gcm}^{-3}$ & $3.7 n g$ & $5.2 n g$ \\
\hline
\end{tabular}

NA - Not Available, AVA: Atomic Vapour Accessory

Limit of detection. Table 3 presents the results for the determination of limit of detection for arsenic under the reaction conditions described earlier. Under these conditions the 95\% confidence limit for detection of arsenic in a single sample was about 5.2ng which is far more superior than achieved with air-acetylene flame methods currently in use (Table 4).

Performance characteristics of air - acetylene AAS methods and the proposed method for determination of arsenic after hydride generation: The values in Table 4 show clearly that the results obtained in this study were significantly better than those achieved with air acetylene flame methods reported by different AAS instrument manufacturers.

As could be observed, the atomic absorption sensitivity and LOD for arsenic using our system were found to be better by a factor of 12 to 16 and 27 to 38 respectively than achieved with airacetylene flames. As a result of the significant improvement in the performance characteristics of our system, environmental levels of arsenic can now be determined at nanogram and subnanogram levels in preference to the conventional air - acetylene method in which the sensitivity and LOD for arsenic (Table 4) are higher than the suspected/environmental levels in the sample. Also the performance characteristics of our manually operated system can be said to compare fairly well with the automated Thermo Jarrel Ash AVA - SH 22 system currently in the market.

In conclusion, a simple and inexpensive system has been developed for the determination of arsenic in a nitrogen - hydrogen entrained air flame after hydride generation atomic absorption spectrophotometry. Because the technique uses the injection of sodium tetrahydroborate (III) solution into the acidified sample solution held in simple pyrex boiling tubes followed by measurement of the average absorption by arsenic atoms generated from the total arsine evolved in a nitrogen - hydrogen entrained air flame, several advantages are offered:

integrating the total absorbance from the total arsine evolved freed the technique from most kinetic interferences associated with peak height measurements.

complete measurement of the evolved hydride within 12 seconds was accomplished because of the design features and operational technique of the hydride generator system.

additional mixing of sample solution in the boiling tube by means of mechanical stirring was eliminated because of the shape of the tube and technique of introducing the sodium tetrahydroborate (III) solution into the very bottom of the tube through a plastic capillary; turbulent mixing of the sample solution that takes place from the bottom of the boiling tube on injection of the sodium tetrahydroborate (III) 
solution facilitates the immediate reduction of the total amount of hydride forming element and stripping of the gaseous hydride from solution by the excess hydrogen produced by decomposition of the sodium tetrahydroborate (III) .

the stripping of the analyte (arsine) from the sample matrix, reduces the potential for chemical interferences in the solution phase.

direct sweeping of the total amount of hydride generated into the AAS by excess hydrogen evolved obviates the need for a complicated apparatus for collecting the hydride and having by-pass systems for sweeping the hydride into the instrument.

operator ability to observe the reaction in its entirety through the boiling tube and to interrupt the determination if excessively intensive foaming threatens carry over of a sample containing organic components into the flame atom cell is guaranteed.

the pyrex glass boiling-tubes used as reduction vessels are convenient to handle and cheap to buy in large quantity while the hydride generation accessory is simple to construct.

the method is sensitive (25ng) and highly reproducible at trace levels having an overall precision of $1.0 \%$ for $0.5 \mu \mathrm{g}$ levels arsenic and a Limit of Detection (95\% confidence) of 5.2ng. The performance characteristics obtained are far more superior than achieved with air-acetylene flame systems currently in the market. In use, the accessory is convenient to operate and fast enabling about forty determinations per hour.

Finally, preliminary work which we hope to report in the near future has already shown that other covalent volatile hydride forming elements of Groups IV, V, and VI can be determined using the hydride generation system .

Also, we have applied the system successfully to determine nanogram quantities of arsenic in environmental and biological sample matrices after appropriate sample treatment.

\section{ACKNOWLEDGEMENT}

One of the authors, Mumuni, A.R thanks the University of Port Harcourt for granting study leave and financial support.

\section{REFERENCES}

Braman, R.S., Justen, L.L, and Foreback, (1972) Direct Volatilization - Spectral Emission Type Detection System for Nanogram Amounts of

Arsenic and Antimony. Anal Chem. 44, (13), 2195.

Fowler, B.A., Ishinishi, N., Tuschiya, K, and Vahter M. (1979) In Friberg, L, Noraberg, G.F. and VouK, V.B. Editors, "Hand book on the Toxicology of Metals” pp 556-557, Elsevier, Amsterdam.

Hernberg, S. (1977) In Hiatt, H.H., Editor, “Origins of Human Cancer”, Cold Spring Habor Laboratories, New York. Vol. 4 pp147-157

Holak, W. (1969). Gas-sampling technique for arsenic determination by atomic absorption spectro photometry. Anal. Chem. 41, 1712.

Nadkarni, R.A. (1982). Applications of hydride generation - atomic absorption spectrophotometry to coal analysis. Anal. Chim. Acta 135,363

Thomson, K.C. and Thomerson (1974) Analyst, 99, 595 